Jose Carlo R. Villanueva, MD Alejandro P. Opulencia, MD

Kenneth Z. Calavera, MD

William L. Lim, MD

Department of Otorhinolaryngology Head and Neck Surgery

University of the East - Ramon Magsaysay

Memorial Medical Center, Inc.

\section{Primary Laryngeal Aspergillosis in a Postpartum Patient}

\begin{abstract}
Objectives: To present a rare case of primary laryngeal aspergillosis manifesting with hoarseness in a seemingly healthy, immunocompetent, postpartum patient and discuss the probable contributing factors leading to this unusual disease process.
\end{abstract}

\section{Methods:}

\author{
Design: Case Report \\ Setting: Tertiary Private University Hospital \\ Subject: One
}

Results: A 28-year-old previously healthy postpartum woman presented with hoarseness of a few weeks duration and recent intake of antibiotics and steroids. Videolaryngoscopy revealed a creamy, exophytic mass overlying both vocal folds. Microscopic examination revealed septated, dichotomously branching hyphae with acute angles characteristic of Aspergillus sp. The patient recovered with anti fungal medications.

Conclusion: The clinical presentation of laryngeal aspergillosis can be very non-specific and should not be disregarded merely on the basis of immune competence. It should be considered, together with other host and environmental factors when a patient responds poorly to conventional treatment. There is a need for quick and accurate diagnosis as the disease responds quite rapidly with appropriate anti fungal medications.

\section{Keywords: Aspergillosis, hoarseness, larynx, postpartum, immunosupression}

Laryngeal aspergillosis is rare and usually involves immunocompromised individuals. ${ }^{1}$ Primary laryngeal aspergillosis is even rarer, with just 21 cases involving immunocompetent individuals in the English literature. ${ }^{1,2}$ We present a case of primary laryngeal aspergillosis manifesting with hoarseness in a seemingly healthy, immunocompetent, postpartum patient and discuss the probable contributing factors leading to this unusual disease process.

\section{CASE REPORT}

A 28-year-old previously well bank teller with no known co-morbidities, presented with fever, cough and colds one day after a cesarean delivery under spinal anesthesia. She was given IV antibiotics which did not relieve her symptoms. Two days postpartum, after several bouts of forceful coughing, she started having hoarseness. Antibiotics was continued for five more days 
and the cough, colds and fever resolved, but her hoarseness persisted. She consulted an otorhinolaryngologist after discharge and was started on Cefuroxime $500 \mathrm{mg}$ and Loratadine $5 \mathrm{mg}+$ Betamethasone $500 \mathrm{mcg}$ twelve-hourly, Esomeprazole $40 \mathrm{mg}$ and Prednisone $50 \mathrm{mg}$ once daily for two weeks with no improvement of her hoarseness. She consulted in our institution, where videolaryngoscopy revealed a creamy exophytic mass overlying both vocal folds. (Figure 1) There was good movement of both true vocal folds but incomplete closure due to the lesion. Chest $x$-ray, complete blood count, serum chemistries and electrolytes were all normal. She underwent direct laryngoscopy with excision of TVF mass under general anesthesia. Tissue sections stained with hematoxylin and eosin revealed acutely angled, dichotomously branching, septated hyphae characteristic of Aspergillus. (Figure 2) An HIV screen was negative, and neck and chest $\mathrm{CT}$ scans were normal. With a diagnosis of laryngeal aspergillosis, she was treated with oral Voriconazole $400 \mathrm{mg}$ once daily for a month. The patient's voice gradually improved with the anti fungal medications, and follow up videolaryngoscopy eventually revealed normal findings. (Figures 3, 4)



Figure 1. Videolaryngoscopy showing creamy curd like exophytic lesion over both vocal folds.

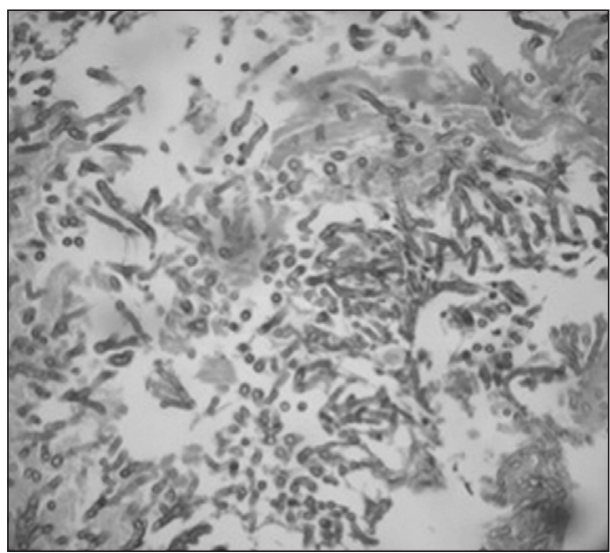

Figure 2. Histopathological section showing acutely angled dichotomously branching septated hyphae characteristic of Aspergillus.

\section{DISCUSSION}

Aspergillus is an inherently non pathogenic or weakly pathogenic fungus that produces a group of opportunistic infections. ${ }^{3}$ It afflicts mostly immunocompromised patients and manifests mainly as a primary pulmonary disease with secondary systemic involvement. ${ }^{4}$ It is due to this nature of the organism that there have been only a few cases of primary laryngeal aspergillosis in immuno-compromised patients ${ }^{5}$ and even less in immuno-competent patients.'

According to Shohami and Levitz, "Aspergillus is a highly aerobic mold with several hundred mold species with worldwide distribution. It is a saprophyte that draws nutrients from soil and vegetation. Spores are easily aerosolized and humans inhale and ingest hundreds of conidia on a daily basis." International and local data has shown that the most frequently isolated airborne mold is Aspergillus. ${ }^{7.8}$

In an immune competent host, ingested or inhaled spores are rapidly cleared by the innate immune response. ${ }^{6}$ Resident and monocytederived macrophages ingest and kill conidia, preventing transition into the invasive hyphal form. ${ }^{6}$

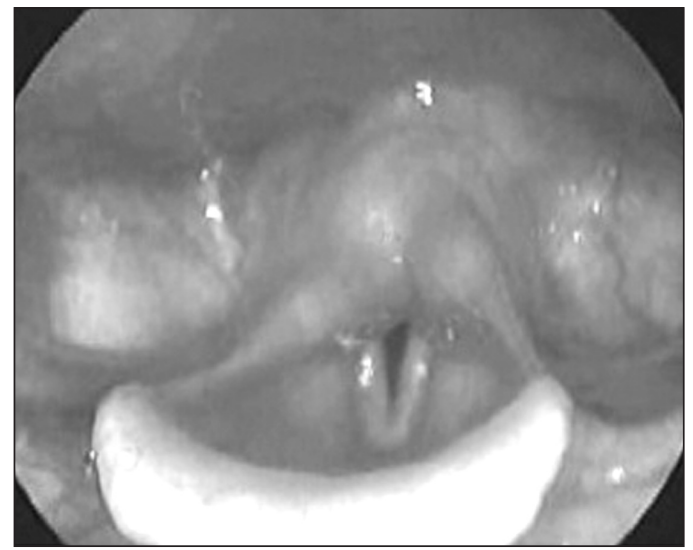

Figure 3. Videolaryngoscopy two weeks post treatment with Voriconazole showing clearing of the lesions.



Figure 4. Normal videolaryngoscopy after ten months. 
The disease form of Aspergillosis most commonly involves the lungs and the tracheo-bronchial tree with subsequent systemic dissemination when host immunity deteriorates. ${ }^{2,4}$ Because of this usual clinical presentation, the fungus has been greatly regarded as an opportunistic infection. It has been reported to have had secondary spread to almost all parts of the human body, including the laryn $x,{ }^{9}$ but the most frequent otolaryngologic sites colonized by Aspergillus are the external auditory canal and the paranasal sinuses. ${ }^{6}$

Primary localization in the larynx is rare specially in patients with intact immune systems. A 20-year literature review of 9,743 patients by Jombo et al. showed the larynx primarily involved only 7 times. Altogether, Liu et al. have listed a total of 20 cases since 1969. Because Aspergillus is weakly pathogenic and is by nature an opportunist, it is worthwhile to look closely into cases such as this, involving healthy individuals and localizing only in the larynx. ${ }^{8}$ Previous case reports have dwelled upon the possible etiologic and contributing factors to the development of the disease in immunocompetent patients. ${ }^{24,5,6,9}$

One factor that has been cited is the use of corticosteroids, particularly inhaled corticosteroids owing to the substantial proportion of particles deposited in the larynx..$^{10}$ Corticosteroids significantly affect the patient's response to the Aspergillus conidia by inhibiting production of reactive oxidant intermediates (a component of intracellular killing) and by depressing neutrophil and macrophage function. ${ }^{2}$

Another potentially contributory factor is the use of broad spectrum antibiotics that could alter local bacterial flora and disturb the ecological balance between bacteria and fungi, allowing the overgrowth of Aspergillus. Several cases of invasive Aspergillosis have been observed in patients receiving multiple antibiotics. ${ }^{11}$

Factors that disrupt the normal mucosal integrity of the larynx have also been implicated. An intact epithelial barrier is an esential protective component. Vocal abuse resulting in disruption of true vocal fold mucosa and vocal fold cysts have been cited as potential aggravating factors. ${ }^{12,13}$

Several of these factors were present in our patient, and may explain how she developed laryngeal Aspergillosis. Although she had no history of vocal abuse, the episodes of forceful coughing could have created areas of disruption or injury in the laryngeal mucosa. She was then given broad spectrum antibiotics and oral steroids, which have all been implicated as possible factors in the development of laryngeal Aspergillosis. ${ }^{2-11}$ Whether her post partum status (marked by decreased natural killer cell function) ${ }^{14}$ was contributory may be a matter for conjecture, as natural killer cells are integral to the innate immune response and play an important role in the body's defense against fungi. All these factors coincidentally occuring simultaneously may have lead to the development of this rare form of primary laryngeal aspergillosis.
To the best of our knowledge, this is the twenty-second reported case of primary laryngeal aspergillosis occurring in an immunocompetent patient in the English literature, the first occurring during the postpartum period, and the first in the Philippines.

The clinical presentation of laryngeal aspergillosis can be very nonspecific and should not be disregarded merely on the basis of immune competence. It should be considered, together with other host and environmental factors (fungal exposure, corticosteroid and antibiotic use, vocal fold injury, recuperative / postpartum states) when a patient responds poorly to conventional treatment. There is a need for quick and accurate diagnosis as the disease responds quite rapidly with appropriate anti fungal medications.

\section{REFERENCES}

1. Liu YC, Zhou SH, Ling L. Aetiological factors contributing to the development of primary laryngeal aspergillosis in immunocompetentpatients. J Med Microbiol. 2010 Oct; 59(Pt 10):1250-1253. DOI 10.1099/jmm.0.021634-0.

2. Doloi PK, Baruah DK, Goswami SC, Pathak GK. Primary aspergillosis of the larynx: acase report. Indian J Otolaryngol Head Neck Surg. 2014 Jan; 66(Suppl 1): 326-8. DOI 10.1007/s12070-0110299-2.

3. Agarwal P,Yadav RP, Upadhyay SN. A new method of detection and differentiation of pathogenic from non-pathogenic Aspergillus species. J Med Microbiol. 2001 Jul; 50(7); 653-654.

4. Shakoor MT, Ayub S, Ayub Z, Mahmood F. Fulminant invasive aspergillosis of the mediastinum in an immunocompetent host: a case report. J Med Case Rep. 2012 Sep 18;6:311.

5. Jombo GTA, Banwat AB, Gyoh SK. Pulmonary and extra pulmonary manifestations of aspergillosis in clinical practice and potential challenges in management: An analysis of literature review. $J$ Clin Med Res. 2010; 2(11): 185-193.

6. Shohami S, Levitz SM. The immune response to fungal infections. Br J Haematol. 2005 Jun; 129(5): 569-582 doi:10.1111/j.1365-2141.2005.05397.

7. Agbayani BT, Reyes AC, Lingao AL, Fontanilla E. A study of airborne fungi in Manila. Acta Medica Philippina. 1968; 5(2) 73-76.

8. Sharma R, Deval R, Priyadarshi V, Gaur SN, Singh VP, Singh AB. Indoor fungal concentration in the homes of allergic/asthmatic children in Delhi, India. Allergy Rhinol. 2011 Jan; 2(1): 21-32. doi: 10.2500/ar.2011.2.0005.

9. Jombo GTA, Banwat AB, Gyoh SK. Pulmonary and extra pulmonary manifestations of aspergillosis in clinical practice and potential challenges in management: An analysis of literature review. $J$ Clin Med Res. 2010; 2(11): 185-193.

10. Fairfax AJ, David V, Douce G. Laryngeal aspergillosis following high dose inhaled fluticasone therapy for asthma. Thorax. 1999 Sep; 54(9):860-861.

11. Nong D, Nong H, Li J, Huang G, Chen Z. Aspergillosis of the larynx: a report of 8 cases. Chin Med J. 1997 Sep; 110(9): 734-736.

12. Mehanna HM, Kuo T, Chaplin J, Taylor G, Morton RP. Fungal laryngitis in immunocompetent patients. J Laryngol Otol. 2004 May; 118(5): 379-381.

13. Wittkopf JC, Connelly S, Hoffman H, Smith R, Robinson R. Infection of true vocal fold cyst with Aspergillus. Otolaryngol Head Neck Surg. 2006 Oct; 135(4): 660-1.

14. Groer M, El-Badri N, Djeu J, Harrington M, Van Eopoel J. Suppression of natural killer cell cytotoxicity in postpartum women. Am J Reprod Immunol. 2010 Mar 1; 63(3): 209-213. doi:10.1111/j.1600-0897.2009.00788.x 